\title{
Research on the Simulation Models of Knowledge Emergence Based on CAS Theory
}

\author{
$\mathrm{Fu} \mathrm{JIN}^{1}$ \\ School of Management \\ Shenyang Normal University, \\ Liaoning, Shenyang, China \\ jinfu8655@163.com \\ (Corresponding Author) \\ Jie JIN $^{3}$ \\ School of Management \\ Shenyang Normal University, \\ Liaoning, Shenyang, China \\ jinjie1111@163.com
}

\author{
Li-Jun ZOU ${ }^{2}$ \\ School of Management \\ Shenyang Normal University, \\ Liaoning, Shenyang, China \\ Jennifer192@163.com \\ Xia WANG ${ }^{4}$ \\ School of Management \\ Shenyang Normal University, \\ Liaoning, Shenyang, China \\ 15702497570@163.com
}

\begin{abstract}
This paper firstly gives the definition of knowledge emergence. Afterwards, based on CAS theory, this paper studies the microcosmic model of multi-Agent and its logic construction of simulation system of macro-level knowledge emergence. This paper reveals evolution rules between the microscopic and macroscopic systems of the knowledge system taking time as a parameter, and gives the applicable conditions of knowledge emergence with CAS simulation methods.
\end{abstract}

Keywords-knowledge emergence; simulation modeling; CAS theory

\section{INTRODUCTION}

The word, "emergence", derives from systematic science. Bertalanffy first introduced the concept of "emergence" in his book named "General System Theory" [1].John Henry Holland argues that "complex science is essentially a discipline about emergence, which studies the basic rules of how to determine a phenomena of knowledge emergence[2]. This paper argues that knowledge and intellectual resource system complies with this law.

Based on above, to understand the concept of knowledge emergence, we take "time" as a dimension of change to make a cognitive explanation of evolution process. We disclose the law of emergence by revealing the relation between microcosmic agents' behavior mechanism and their macro holistic characteristics. The former is a study of a process of phenomenological evolution, and the latter is a study of hierarchical and transitional results.

\section{DEFINITION, CHARACTERISTICS AND RESEARCH FOUNDATION OF KNOWLEDGE EMERGENCE}

\section{A. Definition and Connotation of Knowledge Emergence}

On the definition of the emergence of knowledge, Professor Jin $\mathrm{Fu}$ presents that knowledge emergence is new structure, new patterns and new features emerging from the process of "knowledge self-organization", in which agents with characteristics of "ICAS" is self-organized and controlled. This overall phenomenon of knowledge creation occurs at the macro level of system [4].

Researches show that in the management of intellectual resources, knowledge emergence brings out knowledge creation, and solutions or feasible scheme of unstructured complex problems [4].

\section{B. The Model of Knowledge Emergence Based on CAS Theory}

For the interpretation of knowledge emergence, it is usually with the help of revealing its evolution rules in the interaction of agents from lower level to higher level [5]. In the interaction of agents, multiple adaptive interaction and knowledge sharing generate knowledge evolution, which leads to several emergence results at different levels. These results, as larger agents, have higher level of complex behaviors [6]. The results of knowledge emergence lead macro-system characteristics to change from quantities to qualities [7]

Since 1990s, Holland has begun to establish the theory of Complex Adaptive System, referred to as CAS [2]. Alex Bennet further proposed the Intelligent Complex Adaptive System Theory, 
referred to as ICAS [3]. On this basis, we construct a micro-operation mechanism between agents, which helps to reveal operation mechanism and construct simulation model for the research of knowledge emergence based on CAS theory.

\section{Macro-micro Level Relationship of Intellectual Resource System}

For the interpretation of knowledge emergence of agents, combining with Ryan's research [5], we argue that knowledge emergence is able to capture objective laws of knowledge creation. The principle of knowledge emergence can be a methodology to reveal the law of knowledge creation. The core concept of this principle is to establish an intellectual resource system based on complex science theories [8], which is used to explain and disclose the process of development, methods of identification, and quantitative judgment of knowledge emergence. Knowledge emergence has hierarchical characteristics, which is simplified as macro-state and micro-state [7]. The relationships between levels and relationships among agents in the study of knowledge emergence are shown in Figure 1.

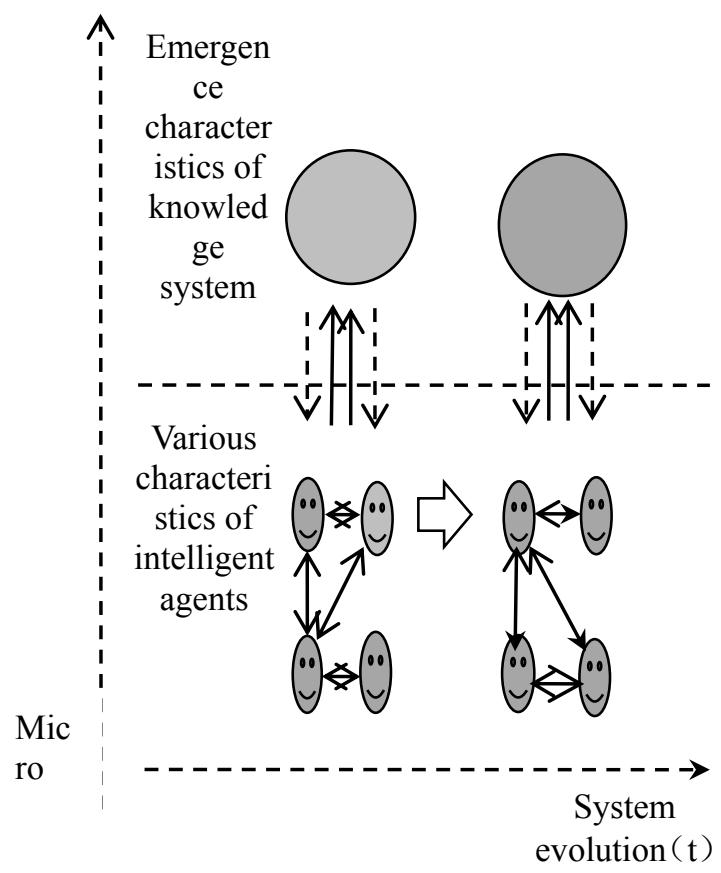

Figure 1. The level and relationships among agents of knowledge emergence

III. RESEARCH AND DESIGN THOUGHT OF KNOWLEDGE EMERGENCE BASED ON SIMULATION TECHNOLOGY.

\section{A. Simulation Technology and Research Basis of Knowledge Emergence}

Computer simulation is simulation model in the process of complex research, which takes computer as the main tool and runs the real systems or imaginary systems in accordance with the similarity principle. Afterwards, it analyzes data from simulation, and comprehensively assesses and predicts actual operational systems and its laws of evolution through comparing simulating results with actual systems [9]. The application of simulation technology in intellectual resource system has the advantages of solving the strategy problems indirectly, simplifying objective world with the construction of model, and so on [10].

The knowledge emergence simulation of the intellectual resource system has the characteristics of systematic quantitative research, which can explain and reveal the process of knowledge emergence and evolution of intelligence system [11]. Through designing the environment of knowledge emergence or adjusting the boundary parameters, we can promote the progress of knowledge emergence, improve the utilization ration of intellectual resources and enhance the efficiency of knowledge creation. These are the core issues of knowledge innovation and technological innovation.

\section{B. Research Basis of Knowledge Emergence Based on Simulation Technology}

Based on similarity theory, CAS simulation establishes models in accordance with certain attributes, relationships or functions of actual systems. We study similarity model to reveal the characteristics of systems [10]. Studies indicate that the intellectual resource system is a kind of special intelligent complex adaptive system (ICAS) [3]. For the intellectual resource system, the experimental tool is a computer software platform; the experimental model is a simplified problem model; the simulation experiment is to run the model and solves problems existed in the model according to the running results and then transforms it into the practical problem solutions [12]. There are several available simulation softwares, such as SWARM, Anylogic, Starlogo, Repast, Ascape, and Vensim [13].

\section{Logical Layer Design of Knowledge Emergence Simulation}

Based on micro-macro analysis principle of CAS knowledge system, we first select the relevant attributes of actual agents based on problems to be studied, and design a micro- model (usually a simple structured model for agents' interaction). Afterwards, we run the model, collect data generated in real time, and integrate these data by 
the macroscopic configuration of agents. Then we analyze the macroscopic characteristics of the intellectual resource system, or observe the macro model of the analysis agent (see Figure 2).

Figure 2. Research framework of knowledge emergence of agents

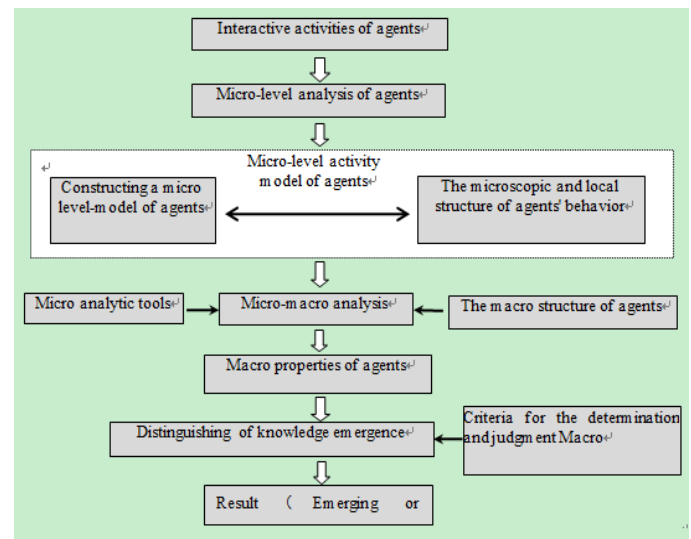

\section{CONTENTS OF CAS KNOWLEDGE EMERGENCE SIMULATION}

\section{A. Macro-micro Analysis Theoretical Basis of Knowledge System}

In order to reveal the laws of knowledge emergence, it sets research contents and themes combining with multi-agent method established in artificial intelligence [11]. Based on Holland's CAS theory, we design agents' macro-micro structure for analysis [2, 14, 15]. From the epistemological analysis, an agent and an intelligent group (Swarm) formed by them reveal the law of knowledge emergence and explain the concept of connotation, features through the representation of their theory and concepts [12].

The general theory of macro-micro analysis mainly consists of the representation of agent, agent theory (AT), and system theory (ST). And the ST-AT consists of macro constrain and micro mechanism. In the intellectual resource system, the micro mechanism refers to the concept of components, and for macro constrain, it refers to the concept of system of components (see figure 3).

Figure 3. A model for knowledge emergence in CAS

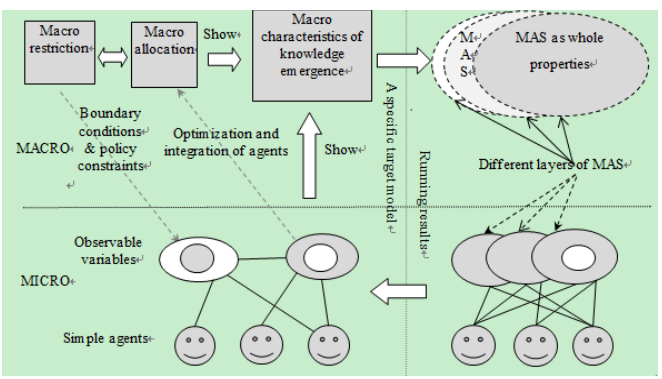

\section{B. The Research Contents of Intellectual Resource} System Simulation

- Establishment of an operational model

From the above research, microscopic behaviors in multi-agent intelligent systems (MAS), behaviors of agents, are clear. What we are going to study is the novel and unpredictable macro characteristics of the system, which are the characteristics of emergence of knowledge system. Therefore, it is a microscopic model that describes agents' certain attributes and an agent is a basic unit in this model.

Overall, a microscopic model utilizes agents' certain attributes and behaviors to reveal the laws of knowledge.

- Revelation and simulation on the macro characteristics of generative rule of knowledge evolution

Through running the model of intellectual resource system, we can intuitively get the macroscopic emergence characteristic of MAS in some aspects [11]. The micro model runs directly to produce intelligent behavior or scattered data of microscopic levels. In order to derive the quantification of the macro knowledge of MAS, it is necessary to synthesize these data. In this process, data are integrated by macro allocation or by visualization technology.

In summary, from the construction of microscopic models, explanation of mechanism and operation to the emergence of macro characteristics of systems, we need constantly to adjust the parameters and amend the model. In macro systems, it is necessary to modify the parameters, to trade-off policy, to remove malicious agents, and to inhibit agents without sharing behavior. More importantly, it is necessary to select heterogeneous (intellectual) resources, to stimulate agents to share knowledge, and to optimize intelligence (resource) configuration.

Based on CAS theory [11], the procedures of simulation modeling are as follows:

- Determine the behavior of agents based on problems to be studied, and select the relevant attributes to design intelligent unit entity, that is, an agent.

- Design micro models according to macro constraints;

- Run the model to observe and collect dynamic evolution data;

- Identify the results of knowledge emergence according to the overall requirements of the system.

It is important to emphasize that the model designed and operated for the results of the pre-existing knowledge is the microscopic model. This micro-model operation mechanism only needs to explain certain attributes or behaviors of agents. 
Meanwhile, it is necessary to identify or select swarm that conforms to boundary conditions and fully explains the entities of knowledge emergence.

Another important content of simulation modeling of knowledge emergence is about macro constraint and configuration. Intelligence resource system reflects the functions of intellectual resource management through macro conditions .Macro constraints refer to constraints that when it comes to select an intellectual agent in process of sampling at system level, we need to consider. For macro allocation, it should embody the related agents of knowledge emergence.

\section{V.CONCLUSION}

According to the researches above, we can draw the following conclusions:

Firstly, based on CAS theory, the key of research on knowledge emergence simulation is the macro-micro analysis of multiple-agents, which helps to disclose the laws of knowledge emergence.

Secondly, the research methods of knowledge emergence based on system simulation obviously has the characteristics of empirical research, which only present a process of evolution and the phenomenon of knowledge creation. In a word, it is difficult to explain the phenomenon of knowledge emergence with accurate mathematical equations.

Thirdly, Comparing with Ikujiro Nonaka's SECI model of knowledge creation, the principle knowledge emergence places much more emphasis on the key role of interpersonal tacit knowledge sharing in the process of knowledge creation and the role of evolution in the process of intelligent agents' adaptive learning.

The advantages of simulation research methods are that comparing with logic and mathematical modeling methods, it is easier to establish and it is more intuitive to reveal intelligent agents' knowledge sharing mechanism. Moreover, it can display the process of knowledge evolution, taking time as a parameter.

However, there is an obvious disadvantage in the simulation research methods of knowledge emergence. There is usually lack of convincing explanation about the inevitability of conditions. In other words, the understanding of inherent knowledge generated from systematic characteristics of knowledge and its evolution mechanism is merely in an intuitive stage, which is difficult to rise to a stage of rational and precision.

To summarize, we will conduct the following researches in the future:

Firstly, we will pay attention to researches on intelligent agents' overall velocity of convergence of knowledge emergence, and researches on identification or judgment of complexity metrics of knowledge emergence.
Secondly, we will pay attention to comprehensive study about knowledge emergence with the combination of simulation research methods of knowledge emergence based on CAS theory with logical methods and mathematical analysis.

\section{REFERENCES}

[1] Mario Bunge. Scientific Materialism [M]. Xiang-lun Zhang and Yu-xing Zhang Transl. Shanghai: Shanghai Translation Press. 1989. pp27-28.

[2] John Henry Holland. Emergence: From Chaos to Order [M], first published in the United States by Oxford University Press. 1998, pp42-45.

[3] Bennet A, Bennet D. Characterizing the next generation knowledge organization: Knowledge \& innovation [J]. Journal of the KMCI. 2000, 1(1), pp8-42.

[4] Jin Fu. Intellectual resources management of knowledge organization [M]. Beijing: Science Press. 2011, pp146-148

[5] Ryan A J. Emergence is coupled to scope, not level: Research Articles [J]. Complexity, 2010, 13(2), pp67-77.

[6] Liu Bei-bei and Jin Fu. Research on MAS model simulation review [J].Computer $\mathrm{CD}$ software and application, 2014 (9), pp15-16

[7] Jin Shi-yao, Huang Hong-bing and Fan Gao-jun. Emergence-oriented research on multi-agent systems and its state of arts $[\mathrm{J}]$ Chinese journal of computers, 2008,31 (6), pp881-895.

[8] Victor Petrenko, Intellectualization Management of Intellectual Resources-Urgent Need of Modern Society [J]. World Scientific News 57(2016), pp674-679.

[9] Fang Mei-qi and Zhang Shu-ren. Complex system modeling and simulation [M]. Beijing: China Renmin University Press, 2011, pp1-11.

[10] Liu H, Abraham A, Clerc M. Chaotic dynamic characteristics in swarm intelligence [J]. Applied Soft Computing Journal, 2007, 7(3), pp1019-1026.

[11] Charles M. Macal, Michael J. North. Tutorial on agent-based modeling and Simulation [C]. In: M. E. Kuhl,N.M. Steger, F. B. Armstrong and J. A. Joines eds. Proceedings of the 2005 Winter Simulation Conference, New York: ACM Press,2005, pp2-15.

[12] Huang Xin-rong. Methodology of the complexity science [M]. Chongqing: Chongqing University Press, 2012, ppp188-201

[13] John H.Holland. Hidden order: how adaptation builds complexity [M]. Zhou Xia-mu and Han transl. Shanghai: Shanghai Scientific and Technical Press. 2000, pp1-15

[14] Jin Fu and Chen Wei. Father of Genetic Algorithms--John Holland and his scientific works [J] Journal of dialectics of nature. 2007, 29(2), pp86-6

[15] Jin Fu and Duan Cheng. A new theory of intellectual resource management in knowledge organizations in the future $[\mathrm{J}]$. Journal of Shenyang Normal University (Social Science Edition). 2012, 36(4), pp58-61.

[16] Luo Ji-gui. Discussion on mechanism for emergence in complex systems [D]. Shanghai: Shanghai University. 2008, pp89-91. 
